\title{
Research on Purchase Order Allocation under Uncertainty of Supply Yan $\mathrm{Li}^{1, \mathrm{a}}$,
}

${ }^{1}$ School of Economics and Management, Jiangsu University of Science and Technology, Zhenjiang Jiangsu China

a18252580511@163.com

Keywords: Supply uncertainty; Order allocation; Purchasing supply chain

\begin{abstract}
Supply uncertainty has a significant impact on the overall supply chain. In this paper, we study the supply chain model of one producer and two suppliers, and establish the expected cost function of the producer and supplier. The change of the total cost of procurement supply chain and the optimal order allocation strategy are analyzed under the condition of independent decision and joint decision. Finally, a numerical example is given to demonstrate the scientific and rationality of the model.
\end{abstract}

\section{供货不确定下采购订单分配研究 \\ 严励 1 , a \\ 1 江苏科技大学经济管理系, 镇江, 江苏, 中国 \\ a18252580511@163.com}

关键词: 供货不确定; 订单分配; 采购供应链

中文摘要：供应商供货不确定对整条采购供应链有着重要影响。本文研究了在交货数量不确 定的情形下, 构建一个生产商和两个供应商的供应链模型, 建立生产商和供应商各个利益主 体的期望成本函数。分析了独立决策和联合决策两种情况下, 采购供应链总成本的变化以及 最优的订单分配策略。最后, 采用某事业部的数据进行系统仿真, 进一步验证了模型的合理 性。

\section{1. 引言}

在装配式制造行业, 例如汽车或电子等实行准时制生产的行业中, 不确定会对整个供应 链中的运作产生巨大影响 ${ }^{[1]}$ 。供应商作为整条采购供应链的主体之一, 是最大的不确定因素。 本文研究准时交货条件下，供应商供货数量不确定的情况。

\section{2. 研究现状}

很多学者从独立决策和联合决策者两个角度来考虑订单分配问题, 并且所构建的模型大 多是单生产商和单供应商组成的单级供应链系统。.Minner 等 ${ }^{[2]}$ 建立了单生产商和单供应商的 系统模型, 分析了随机产出对双方决策优化的影响。Zimmer ${ }^{[3]}$ 研究了在 JIT 交货条件下, 供 应商和生产商完全独立决策以及联合决策下供应链成本的最优化。从目前的研究来看, 大部 分文献以研究一对一的供应链模型为对象, 一对多的研究较少。本文从供应不确定的角度, 主要研究准时交货且供货量不确定的条件下生产商的订货策略以及主供应商的响应策略。 


\section{3. 模型构建}

本文构建的模型如图 1 所示, 主供应商的产能为 $\theta$ ，且 $\theta \in(a, b)$, 其他都是未知的, 只 知道整个供应链的密度函数 $f(\theta)$ 分布函数 $F(\theta)$ 。现在有两种情况, (1)当主供应商接到订单的 时候, $Q \leq \theta N$, 那么次供应商的供应量为零, 意味着主供应商能完全满足订单要求。(2)当 $\theta N<Q$, 主供应商的交货量为 $\theta N$, 次供应商的交货量 $\Delta Q$, 由于量不大, 为了简化模型, 设次 供应商的产能输出是恒满足订单要求的, 则供应商最终的交货量为 $\min (\Delta Q+\theta N, Q)$ 。本文讨 论的就是在第二种情况中, 怎样找到最优的采购量。一方面为了追求自身利益最大化, 建立 生产商成本模型和主供应商成本模型并根据目标函数得出 $Q^{*}$ 和 $\Delta Q^{*}$ 保证各自成本最低; 另方 面, 以整个供应链的总成本最小为目标函数确定 $Q^{* *}$ 和 $\Delta Q^{* *}$ 。表 1 为参数说明。

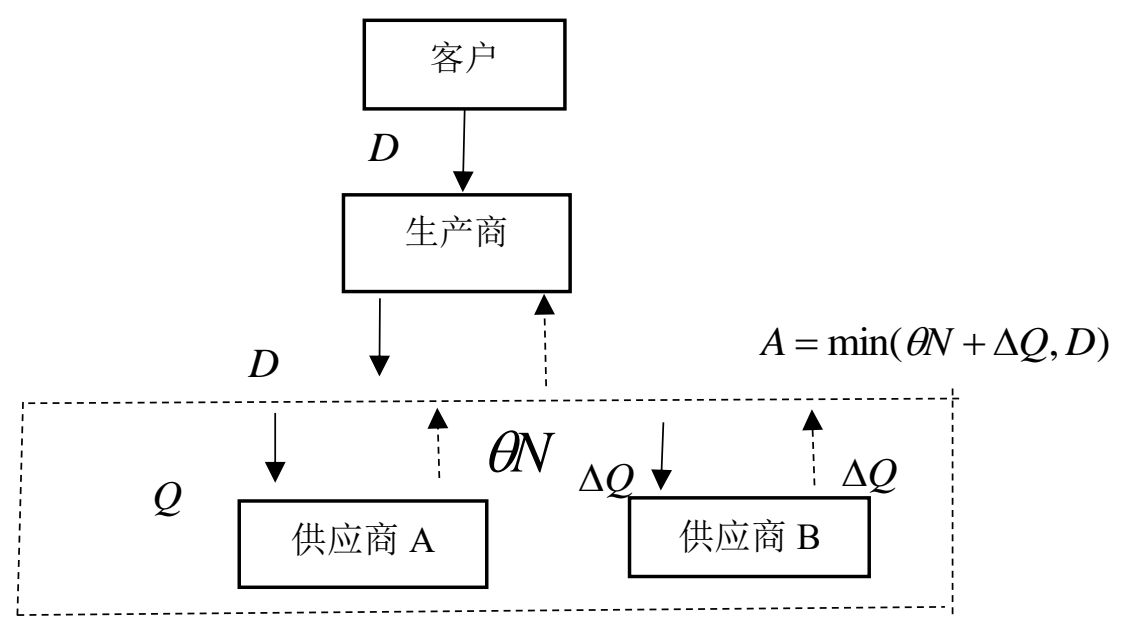

图 1 采购供应链成本模型

表 1 变量与参数说明

\begin{tabular}{|c|l|c|l|}
\hline 变量 & \multicolumn{1}{|c|}{ 说明 } & 变量 & \multicolumn{1}{|c|}{ 说明 } \\
\hline$T C$ & 整条采购供应链的总成本 & $p_{1}$ & 向主供应商的采购价 \\
\hline$C_{p}$ & 生产商的总成本 & $p_{2}$ & 向次供应商的采购价 \\
\hline$C_{s}$ & 主次供应商的总成本 & $\Delta Q$ & 对次供应商的采购量 \\
\hline$D$ & 客户给生产商的需求量 & $s_{2}$ & 周期内供应商的平均库存量 \\
\hline$A$ & 最终交货数量 & $s_{1}$ & 周期内生厂商的安全库存量 \\
\hline$Q$ & 给主供应商的订单 & $U$ & 生产商单位缺货成本 \\
\hline
\end{tabular}




\begin{tabular}{|c|l|c|l|}
\hline$N$ & 主供应商的供应能力 & $p_{3}$ & 供应商单位生产成本 \\
\hline$v_{1}$ & 周期内生产商单位库存成本 & $v_{2}$ & 周期内供应商单位库存成本 \\
\hline$t_{1}$ & 生产商的单位运输成本 & $t_{2}$ & 供应商的单位运输成本 \\
\hline$m_{1}$ & 生产商要求的最小运输量 & $m_{2}$ & 供应商要求的最小运输量 \\
\hline$\alpha$ & 向次供应商采购的单位成本 & $\beta$ & 供应商最优运输批次 \\
\hline$r$ & $\begin{array}{l}\text { 供应商未满足需求而支付的单位惩罚 } \\
\text { 成本 }\end{array}$ & & \\
\hline
\end{tabular}

\section{1 生产商成本模型}

准时采购供应链的最终交货量由主供应商的有效供应量 $\theta N$, 订单数量和 $Q$ 和额外采购 量 $\Delta Q$ 决定的。精益生产要求消除一切不必要的浪费, 因此供应商最终交货的数量应该不大 于生产商要求的数量, $A$ 满足如下的关系。

$$
A=\min \{\theta N+\Delta Q, Q\}
$$

该情况下的生产商的成本模型满足式（2），成本函数如式（3）所示:

$$
C_{p}=P A+U \max (D-A)+V_{1}\left(\frac{S_{1}+A-D}{2}\right)+T_{1}\left(\frac{A}{m_{1}}\right)-R \max (D-A)
$$

$$
\begin{aligned}
& E\left[C_{P}\right]=P_{1} N \int_{a}^{\varphi(\Delta Q, Q)} \theta f(\theta) d \theta+\left(P_{2}+\alpha\right) \int_{\psi(\Delta Q, Q)}^{b} \Delta Q f(\Delta Q) d \Delta Q+U \int_{a}^{\min \{\varphi(\Delta Q), \varphi(\Delta Q, Q)\}}[D-(\theta N+\Delta Q)] f(\theta) d \theta+ \\
& U \int_{\varphi(Q, \Delta Q)}^{b}(D-Q) f(\theta) d \theta+V_{1} \int_{\varphi(\Delta Q)}^{\varphi(Q, \Delta Q)}\left(\frac{s_{1}+\Delta Q+\theta N-D}{2}\right) f(\theta) d \theta+V_{1} \int_{\varphi(Q, \Delta Q)}^{b}\left(\frac{s_{1}+Q-D}{2}\right) f(\theta) d \theta+ \\
& T_{1} \int_{a}^{\varphi(Q, \Delta Q)} \frac{\theta N+\Delta Q}{m_{1}} f(\theta) d \theta+T_{1} \int_{\varphi(Q, \Delta Q)}^{b} \frac{Q}{m_{1}} f(\theta) d \theta \\
& -R \int_{a}^{\varphi(\Delta Q, Q)}[Q-(\theta N+\Delta Q)] f(\theta) d \theta-\frac{1}{2} \int_{a}^{\varphi(\Delta Q, Q)}(D-Q)^{2} f(\theta) d \theta
\end{aligned}
$$

\section{2 主供应商成本模型}

对主供应商来说，它的成本模型如式（4）所示，成本函数如式（5）所示:

$$
C_{S}=P_{3} \min \{\theta N, Q\}+V_{2} \max \left(\frac{S_{2}+\theta N-Q}{2}\right)+T_{2}\left(\frac{\theta N}{m_{2}}\right)+R \max (Q-\theta N)
$$

$$
\begin{gathered}
E\left[C_{S}\right]=p_{3} \int_{a}^{\varphi(\Delta Q, Q)} \theta N f(\theta) d \theta+p_{3} Q \int_{\varphi(\Delta Q, Q)}^{b} f(\theta) d \theta+V_{2} \int_{\varphi(\Delta Q, Q)}^{b}\left(\frac{S_{2}+N}{2}-\theta N\right) f(\theta) d \theta+T_{2} \int_{a}^{\varphi(\Delta Q, Q)} \frac{\theta N}{m_{2}} f(\theta) d \theta \\
+T_{2} \int_{\varphi(\Delta Q, Q)}^{b} \frac{Q}{m_{2}} f(\theta) d \theta+R \int_{a}^{\varphi(\Delta Q, Q)}(Q-\theta N) f(\theta) d \theta-\frac{1}{2} \int_{\varphi(\Delta Q, Q)}^{b}(D-Q)^{2} f(\theta) d \theta
\end{gathered}
$$




\section{4. 独立决策模型}

\section{1 供应商成本模型}

供应链优化的方法中，最为常见的就是从整体和局部或者联合与独立的角度来考虑。局 部优化方法只考虑单个主体自身的利益, 忽视了整个系统的效率。首先, 分析生产商单独决 策的状况。分别对生产商期望成本中的 $Q$ 和 $\Delta Q$ 求二阶偏导数并化简合并同类项

结论 1: 当 $\frac{1}{2} v_{1}>U, \frac{D}{N}=\lambda \neq \frac{a+b}{2}$, 即市场需求占主供应商正常供应能力时, 生产商存在 最优的订单决策 $Q^{*}$ 使得生产商期望总成本最小。

\section{2 供应商成本模型}

供应商作为采购供应链的重要主体之一, 是生产商协调的主要对象和最不可控因素之 一。供应商为了利润最大化从自身利益出发, 作出相应的决策。对式 (5) 的 $Q$ 和 $\Delta Q$ 求二 阶偏导。结论 2: 已知 $\theta \in(a, b)$, 若 $a \neq b$, 则供应商接受生产商的订单量和额外订单量为

$$
\begin{aligned}
& Q^{*}=\frac{\left.D(a+b)+\sqrt{S_{2} V_{2}\left(b^{2}-a^{2}\right.}\right)}{(b-a)} \\
& \Delta Q^{*}=\frac{S_{2} V_{2}(1-R)-R N\left(p_{3}+V_{2}\right)}{(a+b)\left(N V_{2} m_{2}+2 R m_{2}-2 N t_{2}-2 N p_{3} m_{2}\right)}
\end{aligned}
$$

\section{3 联合决策模型}

从系统的角度出发，本文构建的采购供应链的总成本公式如下:

$$
T C=C_{P}+C_{S}
$$

对式（6）的 $Q$ 和 $\Delta Q$ 求二阶偏导。

结论 3: 一定存在最优值 $\left(Q^{* *}, \Delta Q^{* * *}\right)$ 使整条采购供应链总成本达到最优。

\section{5. 仿真分析}

根据事业部的实际情况和数据, 一般情况下, 订货周期是一个月。在单周期内, 供应商 最大的生产能力是 3000 个电池, 生产能力服从 $[0.5,1]$ 均匀分布 (根据数据和实际经验); $\mathrm{B}$ 事业部面临的最终的客户需求是 200 个电池包, 也就是需要 2000 个电池（每个电池包装有 10 个电池)。生产商 对主供应商的采购价格是 1500 元/个; 对次供应商的采购价格是 1800 元; 一个周期内供应商的平均库存量是 300 个, 生产商 安全库存量 100 个; 单周期内如果 没有满足客户的需求导致的缺货成本是 20000 一个; 供应商生产成本是 1000 一个; 供应商 的单位库存成本 50 一个，物流的库存成本是 100 一个; 周期时间内如果供应商没有满足订 单需求必须支付的单位惩罚成本是 1000 一个; 单周期内额外采购寻找其它供应商产生的成 本是 200 一个; 最小起运批量为 10 , 供应商最小的运批量为 50 。已知服从 $[0.5,1]$ 上的均匀 分布, 则的概率密度函数和分布函数是: $f(\theta)=\left\{\begin{array}{l}20<\theta<1 \\ 0\end{array} F(\theta)=\left\{\begin{array}{cc}0 & \theta<0.5 \\ 2 \theta-10.5<\theta<1 & 1<\theta\end{array}\right.\right.$ 
表 2 数据仿真结果

\begin{tabular}{|c|c|c|c|c|c|r|r|}
\hline & & $Q^{*}$ & $\Delta Q^{*}$ & $\theta N^{*}$ & $E\left[C_{p}\right]$ & $E\left[C_{s}\right]$ & $E[T C]$ \\
\hline \multirow{2}{*}{ 单独优化 } & 生产商 & 950 & 1500 & 500 & 3500000 & & 6520000 \\
\cline { 2 - 7 } & 供应商 & 2000 & 50 & 1950 & & 3020000 & \\
\hline 整体优化 & 采购供应链 & 1650 & 200 & 1800 & 2850000 & 1950000 & 4800000 \\
\hline 节约成本 & & & & & 650000 & 1070000 & 1720000 \\
\hline 节约百分比 & & & & & $18.6 \%$ & $35.4 \%$ & $26.4 \%$ \\
\hline
\end{tabular}

通过表 2 中的仿真结果，进行分析可以得出以下结论：

1、联合决策优于单独决策

联合优化决策能够大幅度降低整条采购供应链的成本，从上表中数据可以得到节约的成 本为 $26.4 \%$; 同时，在联合决策情况下，生产商和供应商比单独决策的情况下的成本都下降 了很多, 生产商的成本下降了 $18.6 \%$, 供应商的成本下降了 $35.4 \%$ 。从系统的角度来看, 联 合优化决策能够更好地降低各自成本和整个供应链的总成本。联合决策可以极大地提高采购 供应链的 效率和竞争力。

\section{2、联合决策对供应商更有利}

联合优化决策模型让供应商的期望成本下降 35.4\%，远超过生产商的下降幅度。因此， 联合决策模型更有利于供应商，这就表明供应商更有意愿联合。

\section{References}

[1] LiGuo,ZhangXiang,Ma Shihua. Ordering optimization and coordination in supply chain assembly system under uncertain delivery conditions[J].Computer Integrated Manufacturing Systems,2012,02:369-380.

[2] KELLE P, TRANSCHEL S, MINNER S. Buyer-suppliers cooperation and negotiation support with random yield consideration [J]. International Journal of Production Economics, 2009, 118 (1): 152-159

[3] ZIMMER K. Supply chain coordination with uncertain just-in-time delivery [J]. International Journal of Production Economics, 2002, 77 (1): 1-15 